\title{
New universal 3-3-1 models
}

\author{
William A. Ponce \\ Instituto de Física, Universidad de Antioquia, Medellín, Colombia \\ Artículo de posesión para el ingreso como miembro de número a la \\ Academia Colombiana de Ciencias Exactas, Físicas y Naturales el 6 de Diciembre de 2018
}

\begin{abstract}
We review in a systematic way how anomaly free $S U(3)_{c} \otimes S U(3)_{\mathrm{L}} \otimes U(1)_{X}$ models without exotic electric charges are constructed, using as basis closed sets of fermions which includes each one the particles and antiparticles of the electrically charged fields. Our analysis reproduce not only the known models in the literature, but also shows the existence of two new family independent ones not considered so far. C) 2018. Acad. Colomb. Cienc. Ex. Fis. Nat.
\end{abstract}

Key words: Gauge models; Extensions to the standard model; Gauge anomalies.

Nuevos modelos 3-3-1 universales

\section{Resumen}

Se revisa de manera sistemática como se construyen los modelos sin cargas eléctricas exóticas basados en el grupo gauge local $S U(3)_{\mathrm{c}} \otimes S U(3)_{\mathrm{L}} \otimes U(1)_{X}$, usando como base conjuntos cerrados de fermiones los cuales incluyen las partículas y las antipartículas de los campos eléctricamente cargados. Nuestro análisis no solo reproduce los modelos presentes en la literatura, sino que muestra la existencia de dos modelos nuevos independientes de familias no estudiados a la fecha. (C) 2018. Acad. Colomb. Cienc. Ex. Fis. Nat.

Palabras clave: Modelos gauge; Extensiones al modelo estándar; Anomalías gauge.

\section{Introduction}

The impressive success of the Standard Model (SM) based on the local gauge group $S U(3)_{c} \otimes S U(2)_{L} \otimes U(1)_{Y}$ with the color sector $\mathrm{SU}(3) \mathrm{c}$ confined and the flavor sector $S U(2)_{L}$ $\otimes U(1)_{Y}$ hidden and broken spontaneously by the minimal Higgs mechanism (Donoghue, Golowich \& Holstein, 2014), has not been able enough to provide, among others, explanation for several fundamental issues, among them the masses and mixing angles for both, the quark and the lepton sectors, and the abundance of dark matter and dark energy in the universe. Because of this, many physicists believe that it does not stand for the final theory, representing only an effective model originated from a more fundamental one.

Minimal extensions of the SM arise either by adding new fields, or by enlarging the local gauge group (adding a right handed neutrino field constitute its simples extension, something that ameliorate, but not solve the two problems mentioned above).

Simple extensions of the local gauge group consider an electroweak sector with an extra abelian symmetry $S U(2)_{L}$ $\otimes U(1)_{w} \otimes U(1)_{z}$ (Ponce, 1987), or either the so called left right symmetric model $S U(2)_{L} \otimes S U(2)_{R} \otimes U(1)_{(B-L)}$ and also $S U(3)_{L} \otimes U(1)_{X}$, being the last the one we are going to consider next (Pisano \& Pleitez, 1992; Ponce, et al., 2001).

\section{3-3-1 Models}

In what follows we assume that the gauge group is $S U(3)_{c} \otimes$ $S U(3)_{L} \otimes U(1)_{X}$ (3-3-1 for short) in which the electroweak sector of the standard model $S U(2)_{L} \otimes U(1)_{Y}$ is extended to $S U(3)_{L} \otimes U(1)_{X}$. We also assume that, as in the SM, the color group $S U(3)_{c}$ is vector-like (free of anomalies) and that the left-handed quarks (color triplets) and left-handed leptons (color singlets) transform under the two fundamental representations of $S U(3)_{L}$ (the 3 and $3^{*}$ ).

Two classes of models will show up: universal models where the anomalies cancel in each family as in the SM, and family models where the anomalies cancel by an interplay between the several families.

For the 3-3-1 models, the most general electric charge operator in the extended electroweak sector is

$$
Q=a \lambda_{3}+\frac{1}{\sqrt{3}} b \lambda_{8}+X I_{3}
$$

where $\lambda_{\alpha}, \alpha=1,2, \ldots, 8$ are the Gell-Mann matrices for $S U(3)_{L}$ normalized as $\operatorname{Tr}\left(\lambda_{\alpha} \lambda_{\beta}\right)=2 \delta_{\alpha \beta}$ and $I 3=D g(1,1,1)$ is

\footnotetext{
Corresponding autor:

William A. Ponce, william.ponce@udea.edu.co

Received: October 25, 2018

Accepted: November 19, 2018

Editor: Diógenes Campos Romero
} 
the diagonal $3 \times 3$ unit matrix. $a=1 / 2$ if one assumes that the isospin $S U(2)_{L}$ of the SM is entirely embedded in $S U(3)_{L}$; $b$ is a free parameter which fixes the model and the $X$ values are obtained by anomaly cancellation. $X=0$ for the 8 gauge fields $A_{\mu}^{\alpha}$ of $S U(3)_{L}$, and thus they may be written as (Ponce, et al., 2001):

$$
\sum_{\alpha} \lambda_{\alpha} \mathrm{A}_{\mu}^{\alpha}=\sqrt{2}\left(\begin{array}{ccc}
\mathrm{D}_{1 \mu}^{0} & \mathrm{~W}_{\mu}^{+} & \mathrm{K}_{\mu}^{(\mathrm{b}+1 / 2)} \\
\mathrm{W}_{\mu}^{-} & \mathrm{D}_{2 \mu}^{0} & \mathrm{~K}_{\mu}^{(\mathrm{b}-1 / 2)} \\
\mathrm{K}_{\mu}^{-(\mathrm{b}+1 / 2)} & \mathrm{K}_{\mu}^{-(\mathrm{b}-1 / 2)} & \mathrm{D}_{3 \mu}^{0}
\end{array}\right),
$$

where $D_{1 \mu}^{0}=A_{\mu}^{3} / \sqrt{2}+A_{\mu}^{8} / \sqrt{6}, D_{2 \mu}^{0}=-A_{\mu}^{3} / \sqrt{2}+A_{\mu}^{8} / \sqrt{6}$, and $D_{3 \mu}^{0}=-2 A_{\mu}^{8} / \sqrt{6}$. The upper indices on the gauge bosons stand for the electric charge of the particles, some of them being functions of the $b$ parameter.

\section{The Minimal Model}

Pisano \& Pleitez (1992) shown that, for $b=3 / 2$, the following fermion structure is free of all the gauge anomalies: $\psi_{l L}^{T}=\left(v_{l}^{0}, l^{-}, l^{+}\right)_{L} \sim(1,3,0), Q_{l L}^{T}=\left(d_{i}, u_{i}\right.$, $\left.X_{i}\right)_{L} \sim\left(3,3^{*},-1 / 3\right), Q_{3 L}^{T}=(u 3, d 3, Y) \sim(3,3,2 / 3)$, where $l=e, \mu, \tau$ is a family lepton index, $i=1,2$ for the first two quark families, and the numbers after the similarity sign means 3-3-1 representations. The right handed fields are $u_{a L}^{c} \sim\left(3^{*}, 1,-2 / 3\right), d_{a L}^{c} \sim\left(3^{*}, 1,1 / 3\right)$, $\mathrm{X}_{i L}^{c} \sim\left(3^{*}, 1,4 / 3\right)$ and $\mathrm{Y}_{L}^{c} \sim\left(3^{*}, 1,-5 / 3\right)$, where $a=1$, 2,3 is the quark family index and there are two exotic quarks with electric charge $-4 / 3\left(X_{i}\right)$ and other with electric charge $5 / 3(\mathrm{Y})$.

This version is called minimal in the literature, because it does not make use of exotic leptons including possible right-handed neutrinos.

\section{3-3-1 Models Without Exotic Electric Charges}

If one wishes to avoid exotic electric charges in the fermion and boson sectors as the ones present in the minimal model, one must choose $b=1 / 2$, in Eq. (1) as shown in Ponce, et al. (2001).

To start with, let us consider the following six closed sets of fermions, closed in the sense that they contain the antiparticles of all the charged particles

- $S_{1}=\left[\left(v_{e}^{0}, e^{-}, E^{-}\right) \otimes e^{+} \otimes E+\right]_{L}$ with quantum numbers (1, $3,-2 / 3) ;(1,1,1)$ and $(1,1,1)$ respectively.

- $S_{2}=\left[\left(e^{-}, v_{e}^{0}, N^{0}\right) \otimes e^{+}\right]_{L}$ with quantum numbers $\left(1,3^{*}\right.$, $-1 / 3)$ and $(1,1,1)$ respectively.

- $S_{3}=\left[(d, u, U) \otimes u^{c} \otimes d^{c} \otimes U^{c}\right]_{L}$ with quantum numbers $\left(3,3^{*}, 1 / 3\right) ;\left(3^{*}, 1,-2 / 3\right) ;\left(3^{*}, 1,1 / 3\right)$ and $\left(3^{*}, 1,-2 / 3\right)$ respectively.

- $S_{4}=\left[(u, d, D) \otimes u^{c} \otimes d^{c} \otimes D^{c}\right]_{L}$ with quantum num-bers (3, $3,0) ;\left(3^{*}, 1,-2 / 3\right) ;\left(3^{*}, 1,1 / 3\right)$ and $\left(3^{*}, 1,1 / 3\right)$ respectively.

- $S_{5}=\left[\left(N_{1}^{0}, E_{1}^{+}, e^{+}\right) \otimes E_{1}^{-} \otimes e^{-}\right]_{L}$ with quantum numbers $(1$, $3 *, 2 / 3)(1,1,-1)$, and $(1,1,-1)$ respectively.

- $S_{6}=\left[\left(E_{2}^{+}, N_{2}^{0}, N_{3}^{0}\right) \otimes E_{2}^{-}\right]_{L}$ with quantum numbers $(1,3,1 / 3)$ and $(1,1,-1)$ respectively.
Table 1. Anomalies for 3-3-1 fermion fields structures

\begin{tabular}{lcccccc}
\hline Anomalies & $\boldsymbol{S}_{\mathbf{1}}$ & $\boldsymbol{S}_{\mathbf{2}}$ & $\boldsymbol{S}_{\mathbf{3}}$ & $\boldsymbol{S}_{\mathbf{4}}$ & $\boldsymbol{S}_{\mathbf{5}}$ & $\boldsymbol{S}_{\mathbf{6}}$ \\
\hline$\left[S U(3)_{C}\right]^{2} U(1)_{X}$ & 0 & 0 & 0 & 0 & 0 & 0 \\
{$\left[S U(3)_{L}\right]^{2} U(1)_{X}$} & $-2 / 3$ & $-1 / 3$ & 1 & 0 & $2 / 3$ & $1 / 3$ \\
{$[\mathrm{Grav}]^{2} U(1)_{X}$} & 0 & 0 & 0 & 0 & 0 & 0 \\
{$\left[U(1)_{X}\right]^{3}$} & $10 / 9$ & $8 / 9$ & $-12 / 9$ & $-6 / 9$ & $-10 / 9$ & $-8 / 9$ \\
{$\left[S U(3)_{L}\right]^{3}$} & 1 & -1 & -3 & 3 & -1 & 1 \\
\hline
\end{tabular}

In the former sets $N^{0}$ and $N_{3}^{0}$ can play the role of the right handed neutrino $v_{e}^{0 c}$ in an $\mathrm{SO}(10)$ basis.

Notice that our approach here is different to the one presented in Ponce, et al. (2001), the difference being that only one $S U(3)_{L}$ triplet is used in each set, instead of the composite ones present in the former reference.

The several gauge anomalies calculated for these six sets are proportional to the values quoted in Table 1 (a common constant value or a Kronecker delta can be present in each row in the table). Notice that the anomaly values for $S_{1}, S_{2}$, $S_{3}$ and $S_{4}$ coincide with the ones presented in Ponce, et al. (2001), being the values for $S_{5}$ and $S_{6}$ new results.

Now, if we want to consider only one family of quarks, either the sets $S_{3}$ or $S_{4}$ are enough, but for 3 quark families, one of the following combinations must be used: $3 S_{3}, 3 S_{4}$, $\left(2 S_{3}+S_{4}\right)$ and $\left(S_{3}+2 S_{4}\right)$.

Taking this into account and using the values in Table 1 , we can see that with the particle sets under consideration it is impossible to construct one family (universal) models.

An unrealistic two family model is for example $S_{1}+S_{2}$ $+S_{3}+S_{4}$ [unrealistic because there is strong evidence for at least three families in nature (Donoghue, Golowich $\&$ Holstein, 2014)].

Right from Table 1 it is simple to read the following two three family models free of anomalies:

- Model A: $3 S_{2}+S_{3}+2 S_{4}$.

- Model B: $3 S_{1}+2 S_{3}+S_{4}$,

two well known models in the literature; A being named as a "Model with right-handed neutrinos" (Foot, et al., 1994; Benavides, et al., 2009; Montero, et al., 1993) and B named as a "Model with exotic charged leptons" (Özer, 1996; Ponce \& Zapata, 2006; Salazar, et al., 2007).

The strategy now is to use the lepton sets $S_{1}, S_{2}, S_{5}$ and $S_{6}$ to build non vector-like new sets of leptons. Notice that Vector-like sets as for example $S_{1}+S_{5}$ and $S_{2}+S_{6}$ are free of anomalies and not suitable for constructing realistic models due to the non zero anomalies in the quark sector [Vectorlike sets are free of anomalies by definition (Donoghue, Golowich \& Holstein, 2014)].

The new lepton sets we want to consider are now:

- $S_{7}=\left[\left(e^{-}, v_{e}^{0}, N_{1}^{0}\right) \otimes\left(N_{2}^{0}, E^{+}, e^{+}\right) \otimes E^{-}\right]_{L}$ with quantum numbers $\left(1,3^{*},-1 / 3\right) ;\left(1,3^{*}, 2 / 3\right)$ and $(1,1,-1)$ respectively. 
- $\left.S_{8}=\left[v_{e}^{0}, e^{-}, E^{-}\right) \otimes\left(E+, N_{1}^{0}, N_{2}^{0}\right) \otimes \mathrm{e}^{+}\right]_{L}$ with quantum numbers $(1,3,-2 / 3),(1,3,1 / 3)$ and $(1,1,1)$ respectively.

- $S_{9}=\left[\left(e^{-}, v e, N_{1}^{0}\right) \otimes\left(E-, N_{2}^{0}, N_{3}^{0}\right) \otimes\left(N_{4}^{0}, E+, e^{+}\right)\right]_{L}$ with quantum numbers $\left(1,3^{*},-1 / 3\right) ;\left(1,3^{*},-1 / 3\right)$ and $\left(1,3^{*}\right.$, $2 / 3$ ) respectively.

- $S_{10}=\left[v e, e^{-}, E_{1}^{-}\right) \otimes\left(E_{2}^{+}, N_{1}^{0}, N_{2}^{0}\right) \otimes e^{+} \otimes\left(N_{3}^{0}, E_{2}^{-}, E_{3}^{-}\right) \otimes$ $\left.E_{1}^{+} \otimes E_{3}^{+}\right]_{L}$ with quantum numbers $(1,3,-2 / 3) ;(1,3,1 / 3) ;$ (111); (1, 3, -2/3); (111); (111), and (111) respectively.

The anomalies for these new lepton sets are given in Table 2. Again, the $S U(2)_{L}$ singlets $N_{1}^{0}$ in $S_{7}$ and $S_{9}, N_{2}^{0}$ in $S_{8}$ and $S_{10}$ and $N_{3}^{0}$ in $S_{9}$ can play the role of the right handed neutrino field $v_{e}^{0 c}$.

Notice that again $S_{7}+S_{8}$ is Vector-like and because of this free of anomalies.

With the anomaly values in Table 1 and Table 2 new one family and three family models can be constructed. As a matter of fact, the following three family models show up:

- Model C: with unique lepton generation one $S_{1}+S_{2}+$ $S_{3}+2 S_{4}+S_{9}$

- Model D: with unique lepton generation two $S_{1}+S_{2}+$ $2 S_{3}+S_{4}+S_{10}$

- Model E: hybrid one $S_{3}+2 S_{4}+2 S_{9}+S_{10}$

- Model F: hybrid two $2 S_{3}+S_{4}+S_{9}+2 S_{10}$

The phenomenology for the lepton sector of the two models with unique lepton generation (models $\mathrm{C}$ an $\mathrm{D}$ ) has been presented by Anderson \& Sher (2005). The phenomenology of the two hybrid models $\mathrm{E}$ and $\mathrm{F}$ have not been presented in the literature as far as we know.

Also, the following four one family models (universal) are readily constructed:

- Model G: carbon copy one $S_{4}+S_{9}$

- Model H: carbon copy two $S_{3}+S_{10}$

- Model I: carbon copy three $2 S_{2}+S_{4}+S_{5}$

- Model J: carbon copy four $2 S_{1}+S_{3}+S_{6}$

According to Sanchez, et al. (2001), the fermion structure of model $\mathrm{G}$ can be embedded in the unification group $\mathrm{E}(6)$, with some phenomenology of this structure allready presented in the same reference.

In a similar way, the fermion structure of model $\mathrm{H}$ can

be embedded in the gauge group $S U(6) \otimes U(1)$ as presented by Martinez, et al. (2001).

As can be seen, the first eight models A....... H have been already reported in the literature (Ponce, et al., 2001); but models I and $\mathrm{J}$ are new ones!

The name "Carbon Copy" we adopted is due to the fact that realistic, 3 family models, can be constructed just by repeating three times the corresponding fermion structures, just as in the SM.

All the models presented here can also be enlarged by adding to each one of them an extra vector like structure $\left(S_{1}\right.$ $+S_{5}$ and $/$ or $S_{2}+S_{6}$ ).

The fermion structures studied in this section are so rich, that they can be used to construct non universal models
Table 2. Anomalies for the 3-3-1 non Vector-Like lepton fields structures

\begin{tabular}{lcccc}
\hline Anomalies & $\boldsymbol{S}_{\mathbf{7}}$ & $\boldsymbol{S}_{\mathbf{8}}$ & $\boldsymbol{S}_{\mathbf{9}}$ & $\boldsymbol{S}_{\mathbf{1 0}}$ \\
\hline$\left[S U(3)_{C}\right]^{2} U(1)_{X}$ & 0 & 0 & 0 & 0 \\
{$\left[S U(3)_{L}\right]^{2} U(1)_{X}$} & $1 / 3$ & $-1 / 3$ & 0 & -1 \\
{$[\operatorname{Grav}]^{2} U(1)_{X}$} & 0 & 0 & 0 & 0 \\
{$\left[U(1)_{X}\right]^{3}$} & $-2 / 9$ & $2 / 9$ & $6 / 9$ & $12 / 9$ \\
{$\left[S U(3)_{L}\right]^{3}$} & -2 & 2 & -3 & 3 \\
\hline
\end{tabular}

for several families (two, three, four, five, etc.). In particular, four family models using these structures were presented for the first time by Ponce, et al. (2010).

To conclude this section let us mention that the extension of this analysis to non universal three family models (work in progress) can be used to explain the experimental anomalies reported recently in the Large Hadron Collider.

\section{The 3-3-1 model with righthanded neutrinos}

To make explicit our notation, let us present here the Fermion structure of the most popular of the models analyzed here, the so called "model with right-handed neutrinos", introduced in the literature for the first time by Montero, et al. (1993) and Foot, et al. (1994). This model has the following 3-3-1 anomaly free fermion structure:

$$
\begin{aligned}
\psi_{l L}^{T} & =\left(l^{-}, v_{l}^{0}, N_{l}^{0}\right)_{L} \sim\left(1,3^{*},-1 / 3\right), l_{L}^{+} \sim(1,1,1), \\
Q_{i L}^{T} & =\left(u_{i}, d_{i}, D_{i}\right)_{L} \sim(3,3,0), \\
Q_{3 L}^{T} & =\left(d_{3}, u_{3}, U\right)_{L} \sim\left(3,3^{*}, 1 / 3\right),
\end{aligned}
$$

where $l=e, \mu, \tau$ is a family lepton index, $N_{l L}^{0}$ stands for electrically neutral Weyl states, and $i=1,2$ for the first two quark families. The right handed quark fields are

$$
\begin{aligned}
u_{a L}^{c} & \sim\left(3^{*}, 1,-2 / 3\right), \quad d_{a L}^{c} \sim\left(3^{*}, 1,1 / 3\right), \\
D_{i L}^{c} & \sim\left(3^{*}, 1,1 / 3\right), \quad U_{L}^{c} \sim\left(3^{*}, 1,-2 / 3\right),
\end{aligned}
$$

where again $a=1,2,3$ is the quark family index. Once more, there are two exotic quarks with electric charge $-1 / 3\left(D_{i}\right)$ and other with electric charge $2 / 3(U)$.

The extended phenomenology studies done for this model shows that $N_{l}^{0}, l=e, \mu, \tau$ can be identified with the right-handed neutrinos $v_{l}^{0 c}$, which belong to the same 3-3-1 representation of the neutrino fields; so, lepton number is not a good quantum number for this particular model and it is not conserved (Chang \& Long, 2006).

\section{New universal 3-3-1 models}

Let us see the fermion structure of these two new models which appears from our systematic analysis:

Model I. The particle content for this model is the following:

$$
\begin{aligned}
& {\left[\left(e^{-}, v_{e}^{0}, N^{0}\right) \oplus e^{+} \oplus\left(E_{1}^{-}, N_{1}^{0}, N_{2}^{0}\right) \oplus E_{1}^{+}\right]_{L} \oplus} \\
& {\left[\left(N_{3}^{0}, E_{2}^{+}, E_{3}^{+}\right) \oplus E_{2}^{-} \oplus E_{3}^{-}\right]_{L} \oplus} \\
& {\left[(u, d, D) \oplus u^{c} \oplus d^{c} \oplus D^{c}{ }_{L}\right.}
\end{aligned}
$$


A model with an extra down quark, three exotic electrons and five neutral Weyl states in each family.

Model $\boldsymbol{J}$. The particle content for this model is the following

$$
\begin{aligned}
& {\left[\left(v_{e}^{0}, e^{-}, E_{1}^{-}\right) \oplus e^{+} \oplus E_{1}^{+} \oplus\left(N_{1}^{0}, E_{2}^{-}, E_{3}^{-}\right)\right]_{L} \oplus} \\
& {\left[E_{2}^{+} \oplus E_{3}^{+} \oplus\left(E_{4}^{+}, N_{2}^{0}, N_{3}^{0}\right) \oplus E_{4}^{-}\right]_{L} \oplus} \\
& {\left[(d, u, U) \oplus u^{c} \oplus d^{c} \oplus U^{c}\right]_{L}}
\end{aligned}
$$

A model with an extra up quark, four exotic electrons and four neutral Weyl states in each family.

The phenomenology for these two new models is being carried through right now by our research group and will be presented elsewhere.

\section{Summary}

In this paper we have done a systematic analysis in order to construct 3-3-1 models without exotic electric charges; ten models appear, eight of them already considered in our previous analysis (Ponce, et al., 2001) and two new ones which we believe deserve special attention.

The next question is how to break the 3-3-1 gauge symmetry and provide with masses to the fermion fields in each model. The answer for this is a matter not settled yet. In Ponce, et al. (2003) the minimal set of Higgs scalars able to break the symmetry is presented, something called in the literature as the economical 3-3-1 model; unfortunately, it is not clear yet if this minimal set provides with masses to all the fermion fields in each model, something which is very unlike (Montero \& Sánchez-Vega, 2015). A more realistic set of Higgs scalars able to break the symmetry and to provide masses to the fermion fields has been studied in detail (Ponce \& Giraldo, 2001; Ponce, et al., 2009).

A very attractive fact about the economical 3-3-1 structure is that in some versions of it, the troublesome Majoron is gauged away and is not present en the particle spectrum (Benavides, et al., 2015).

Finally, let us mention that the phenomenology for the two new models presented is under consideration right now, and the results will be presented else where.

\section{References}

Anderson, D.L. and Sher, M. (2005). Phys. Rev. D 72, 095014.

Benavides, R.H., Epele, L.N, Fanchiotti, H., García Canal, C., and Ponce, W.A. (2015). Adv. in High Energy Physics, 813129.

Benavides, R.H., Giraldo, Y. and Ponce, W.A. (2009). Phys. Rev. D 80, 113009.

Chang, D. and Long, H.N. (2006). Phys. Rev. D 73, 053006.

Donoghue, J.F., Golowich, E. and Holstein, B.R. (2014). Dynamics of the standard model, Second Edition. Cambridge, England: Cambridge University Press. This reference is an excellent compendium for the SM.

Foot, R., Long, H.N. and Tran, T.A. (1994). Phys. Rev. D50, R34.

Martinez, R. Ponce, W.A. and Sánchez, L.A. (2002). Phys. Rev. D 65, 055013.

Montero, J.C., Pisano, F. and Pleitez, V. (1993). Phys. Rev. D47, 2918.

Montero, J.C. and Sánchez-Vega, B.L. (2015). Phys. Rev. D 91, 037302.

Özer, M. (1996). Phys. Rev. D 54, 1143.

Pisano, F. and Pleitez, V. (1992). Phys. Rev. D 28, 540.

Ponce, W.A. (1987). Phys. Rev. D 36, 962.

Ponce, W.A., Benavides, R.H. and Giraldo, Y. (2010). Phys. Rev. D 82, 013004.

Ponce, W.A., Flórez, J.B., and Sánchez, L.A. (2001). Int. J. Mod. Phys. A 17, 643.

Ponce, W.A. and Giraldo, Y. (2011). The European Physical Journal C71, 1693.

Ponce, W.A., Giraldo, Y. and Sánchez, L.A. (2009). The European Physical Journal C63, 461.

Ponce, W. A., Giraldo, Y. and Sánchez, L.A. (2003). Phys. Rev. D 67, 075001 .

Ponce, W.A. and Zapata, O. (2006). Phys. Rev. D 74, 093007.

Salazar, J.C., Ponce, W.A. and Gutiérrez, D. (2007). Phys. Rev. D 75, 075016.

Sánchez, L.A, Ponce, W.A. and Martinez, R. (2001). Phys. Rev. D 64, 075013. 